\title{
BMJ Open Adherence to COVID-19 protective behaviours in India from May to December 2020: evidence from a nationally representative longitudinal survey
}

\author{
Simone Schaner (D , , ${ }^{1,2}$ Natalie Theys, ${ }^{1}$ Marco Angrisani, ${ }^{1,2}$ Joyita Banerjee, ${ }^{3}$ \\ Pranali Yogiraj Khobragade, ${ }^{1}$ Sarah Petrosyan, ${ }^{1}$ Arunika Agarwal, ${ }^{4}$ Sandy Chien, ${ }^{1}$ \\ Bas Weerman, ${ }^{1}$ Avinash Chakrawarty, ${ }^{3}$ Prasun Chatterjee, ${ }^{3}$ Nirupam Madaan, ${ }^{5}$ \\ David Bloom, ${ }^{4}$ Jinkook Lee, ${ }^{1,2}$ Aparajit Ballav Dey ${ }^{3}$
}

To cite: Schaner S, Theys N, Angrisani M, et al. Adherence to COVID-19 protective behaviours in India from May to December 2020: evidence from a nationally representative longitudinal survey. BMJ Open 2022;12:e058065. doi:10.1136/ bmjopen-2021-058065

- Prepublication history and additional supplemental material for this paper are available online. To view these files, please visit the journal online (http://dx.doi.org/10.1136/ bmjopen-2021-058065).

Received 05 October 2021 Accepted 10 January 2022

Check for updates

(c) Author(s) (or their employer(s)) 2022. Re-use permitted under CC BY-NC. No commercial re-use. See rights and permissions. Published by BMJ.

For numbered affiliations see end of article.

Correspondence to Dr Simone Schaner; schaner@usc.edu

\section{ABSTRACT}

Objectives Since the onset of the COVID-19 pandemic, behavioural interventions to reduce disease transmission have been central to public health policy worldwide. Sustaining individual protective behaviour is especially important in low-income and middle-income settings, where health systems have fewer resources and access to vaccination is limited. This study seeks to assess time trends in COVID-19 protective behaviour in India.

Design Nationally representative, panel-based, longitudinal study.

Setting We conducted a panel survey of Indian households to understand how the adoption of COVID-19 protective behaviours has changed over time. Our data span peaks and valleys of disease transmission over MayDecember 2020.

Participants Respondents included 3719 adults from 1766 Indian households enrolled in the Harmonised Diagnostic Assessment of Dementia for the Longitudinal Ageing Study in India.

Analysis We used ordinary least squares regression analysis to quantify time trends in protective behaviours. Results We find a 30.6 percentage point $(95 \% \mathrm{Cl}(26.7$ to 34.5$) ; p<0.01$ ) decline in protective behaviours related to social distancing over the observation period. Mask wearing and handwashing, in contrast, decreased by only 4.3 percentage points $(95 \% \mathrm{Cl}(0.97$ to 7.6$) ; p<0.05)$ from a high base. Our conclusions are unchanged after adjusting for recorded COVID-19 caseload and nationwide COVID-19 containment policy; we also observe significant declines across socioeconomic strata spanning age, gender, education and urbanicity.

Conclusion We argue that these changes reflect, at least in part, 'COVID-19 fatigue,' where adherence to social distancing becomes more difficult over time irrespective of the surrounding disease environment.

\section{INTRODUCTION}

Throughout the COVID-19 pandemic, governments around the world have implemented non-pharmacological policies aimed at blunting disease spread. Although policies

\section{Strengths and limitations of this study}

- Our study leverages data from a nationally representative panel survey in India to study changes in COVID-19 protective behaviour between May and December 2020.

- We link our survey data to contextual data measuring COVID-19 caseload and national COVID-19 policy, allowing us to assess the robustness of our main results to the disease and policy environments.

- We study how time trends in protective behaviour vary among key demographic groups.

- Our surveys were conducted over the phone, which runs the risk of under-representing India's most socioeconomically disadvantaged households.

- Our measures of protective behaviour do not capture frequency or intensity within the lookback period.

have shifted over time-changing in scope and stringency ${ }^{1}$ - a common aim has been to drastically reduce the mobility of, and social contact among, people. Critical in assessing the efficacy of these policies, and thus how to improve them, is understanding how distancing behaviour changes or persists in the face of easing restrictions and evolving disease environments.

Much of the existing research in this space leverages cellphone data (most notably, open-source mobility datasets like Google's COVID-19 Community Mobility Reports) to characterise movement patterns. ${ }^{2-5}$ Cellphonebased mobility data, however, fail to fully capture important facets of behaviour that matter for disease transmission. For example, such data cannot record maintaining physical distance, avoiding large crowds or wearing masks, all of which are common components of containment policies, and evidence suggests that adherence to these types of behaviours 
may be more useful for forecasting disease trajectory than measurements of movement alone. ${ }^{6}$ In addition, macrolevel mobility analyses that rely on data captured from mobile phones run the risk of concealing deep disparities in both adherence and impact.

These data limitations resonate particularly in lowincome and middle-income countries (LMICs), where smartphone usage remains far from universal and survey data remain scarce. Understanding the ability of LMIC populations to maintain social-distancing practices over an extended period of time is especially pressing given (1) concerns that COVID-19 will disproportionately harm those living in LMICs, ${ }^{8-10}$ and (2) the fact that LMICs continue to lag in vaccine acquisition and administration $^{11}$ and, thus, may need to rely predominantly on nonpharmacological interventions for an extended period of time.

Understanding trends in distancing and other protective behaviours in India is significant, as it is the world's second largest LMIC and its population is uniquely vulnerable given the nation's high population density, large share of multigenerational households and substantial population of individuals with COVID-19 risk factors like hypertension and diabetes. ${ }^{12}$ This vulnerability was evident as the country experienced one of the world's deadliest waves of COVID19, which began in April 2021. Various reasons have been cited for this resurgence, including the emergence of more contagious variants, a poorly coordinated, too-lax containment approach left in large part up to states ${ }^{13}$ and a lagging vaccine campaign. ${ }^{14}$ Critically, little rigorous data exist on the extent to which distancing behaviours were adopted and retained during the initial lockdown in 2020, or on how those behaviours changed during subsequent periods of reopening. Such insights could prove crucial to understanding the differing contexts of India's COVID-19 waves and their severity.

To help fill this information gap, we designed and fielded a nationally representative, high-frequency phone survey of Indian households to monitor knowledge, attitudes and behaviours related to COVID-19. The survey, which also tracks the economic and health conditions of households, has been conducted bimonthly since India's nationwide lockdown in March 2020. This initiative allows us to construct representative estimates of COVID-19 protective behaviours in India over time and to characterise how these behaviours differ across key socioeconomic groups. Unique in its scope, detail and coverage, our study is a novel contribution to the existing literature, which has focused on adherence to COVID-19 protective behaviours in specific regions ${ }^{1516}$ or on specific populations, ${ }^{17}$ or used cellphone data to understand broad trends in mobility patterns. ${ }^{18-20}$

\section{METHODS}

\section{Background: COVID-19 containment in India}

India's central government reacted to the hastening spread of COVID-19 with an initial lockdown on 25 March 2020, implemented with less than 24 hours' notice.
Although initially meant to be in effect for 1 week, the directive was subsequently extended four times and ultimately lasted more than 2 months. The restrictions immediately halted public transportation, mandated mask wearing, closed all non-essential businesses and banned many social gatherings.

After the national lockdown ended on 31 May 2020, the central government initiated reopening through various 'unlock' phases while ceding future control over lockdowns and closures to individual states. Although decisions to reopen economically varied across geographies, protective behaviours-like maintaining social distance, avoiding unnecessary travel and wearing masksremained widely encouraged; for a more in-depth look at India's initial lockdown timeline, refer to previous work. ${ }^{21}$ During the unlock phases, caseloads remained low; however, the country subsequently experienced a spike in cases late in the summer and early fall of 2020. Following a lull in cases during the winter, infections again began to grow at an alarming rate starting in March 2021; by 15 April 2021, India had clearly entered a second COVID-19 surge unparalleled in the rest of the world, with nearly every state reporting a rapid growth in infections. ${ }^{22}$ Online supplemental figure $\mathrm{S} 1$ graphs the Indian COVID-19 caseload and an index capturing the stringency of India's national policy response against our survey waves, described in detail as follows.

\section{The data}

We leveraged an existing study called the Harmonised Diagnostic Assessment of Dementia for the Longitudinal Ageing Study in India (LASI-DAD), a nationally representative study that aims to understand patterns in cognition and dementia among older Indians. ${ }^{23}$ Out of the 3316 LASI-DAD households, we contacted all 2704 who had valid phone numbers in May 2020 to invite them to participate in a bimonthly phone survey that covered various topics related to household well-being and COVID-19related knowledge, attitudes and behaviour. All households contained at least one individual over the age of 60 .

The analyses presented in this paper use four waves of survey data: Wave 1 took place from May 5 through 25 June 2020; Wave 2 took place from 7 July through 26 August 2020; Wave 3 took place from 7 September through 23 October 2020; and Wave 4 took place from 9 November 2020 through 4 January 2021. Most of the Wave 1 survey occurred while the nation was still under the initial mandatory lockdown. Additional waves of data collection are scheduled to continue through December 2021.

During Wave 1, two randomly selected household members over the age of 18 (one male and one female, if possible) were invited to participate. (Names were drawn from a household roster collected as part of the earlier LASI-DAD survey.) In subsequent waves, we aimed to maintain continuity in the interviewed household members: if an enrolled individual could not be reached, the enumerator scheduled an appointment for 
a future time; if this follow-up was unsuccessful, another adult household member was selected to participate in that wave instead. In Wave 3, we attempted to enrol all primary LASI-DAD respondents (individuals over the age of 60 who had participated in prior in-person waves of data collection during 2017 through 2019). Each wave targeted all individuals who had ever participated in a past wave. As a result, some households have up to four individuals interviewed in some waves. By collecting these data at a relatively high frequency, we were able to capture behaviour changes made in the face of fast changing and dynamic policy and disease environments. The panel nature of our data also allows us to estimate within-person changes in distancing behaviour, a useful way of ensuring our results are not driven by changes in sample composition/selective survey response.

The final sample includes 3719 individuals from 1766 households; 1019 of these individuals and 665 of the households participated in all four waves (refer to online supplemental figure $\mathrm{S} 2$ for a breakdown of the final sample). Prior to each wave of data collection, all participants were required to provide informed, verbal consent, following protocols as approved by the Institutional Review Board (IRB) at both the University of Southern California (USC; study number UP-20-00277) and the All India Institute of Medical Sciences (AIIMS; study number RP-29/2020). We use sample weights to ensure estimates are nationally representative. The Weight construction section provides additional detail. Online supplemental table S1 provides summary statistics for our sample; column 5 includes weighted statistics for individuals who participated in all four waves, while column 6 contains the unweighted statistics. Our sample over-represents older individuals (60+), as expected given our initial sample and the focus on interviewing LASI-DAD respondents. The sample also over-represents those with higher levels of education, which may reflect the fact that our survey is phone-based and phone ownership is correlated with higher education and socioeconomic status in India. The analyses herein employ weights, so they can be interpreted as nationally representative, and include all individuals from each wave. Online supplemental figure S3 shows the geographical scope of our sample. Although our study sample is mostly rural, reflecting the population distribution of the country, we also cover some of India's megacities, including Mumbai and Delhi, which to date have experienced the country's worst COVID-19 outbreaks. ${ }^{24} 25$

We use information on district of residence and survey date to attach contextual data on COVID-19 caseload in the preceding 2 weeks to each interview. Caseload, quantified as the daily number of new confirmed cases, was obtained from Covid19india.org, a crowd-sourced initiative that compiles daily statistics on COVID-19. (Covid19 india.org collates state-level and district-level data from official bulletins and Twitter handles. Data are validated by a group of volunteers before release. For a full list of their source sites, refer to Covid19india.org.) Due to delays in the processing and reporting of test results, we chose to smooth these estimates by taking a caseload average across the 14 days prior to the survey date. Finally, using total district-level population estimates from the 2011 Census of India, we calculated the number of cases per 10000 . District-level caseload statistics were not available in Assam, Telangana and Delhi; thus, state-level statistics were used for these states. (Delhi is classified as a union territory rather than a state. However, we use the term 'state' to refer to both states and union territories throughout the text to simplify exposition.)

Finally, we account for national COVID-19 containment policy by using the 'Government Response Index' from the Oxford COVID-19 Government Response Tracker, which aggregates indicators of containment and health policy (such as school and workplace closings, restrictions on movement), economic policy (income support and debt relief) and health system policy (including facial covering policy and contract tracing). The index ranges from 0 to 100 , with higher values indicating more aggressive policy action. Additional detail on index components and methodology is available in previous work. ${ }^{26}$ We use data on survey date to attach the average value of the index in the 2 weeks prior to interview onto each survey record.

\section{Patient and public involvement}

Survey respondents were not directly involved in the study design, including the development of research questions, survey design or recruitment. There are no plans to directly disseminate the results to survey participants.

\section{Measures of COVID-19 protective behaviour}

Non-pharmacological measures to curb the spread of COVID-19 have used a combination of mandates and public health messaging to minimise social contact across households and highlight the importance of personal hygiene. To understand the extent to which individual behaviours are aligned with these initiatives, we group behaviours tracked in our survey into three broad categories: market-based distancing behaviours, protective behaviours and social-distancing behaviours. The recall period for each individual behaviour is 7 days. Market-based behaviours include activities that may not be fully discretionary-that is, they may reflect maintaining a person's livelihood, either through work or buying food. These activities include attending a gathering with 10 or more people, having close contact (described to respondents as 'two arms' lengths') with non-household members, travelling for work and going shopping. We classify an individual as 'market distancing' if she/he does not report any of the aforementioned behaviours. The second group is protective behaviours, which includes the two main hygiene behaviours consistently cited as key mechanisms for decreasing disease spread: handwashing and wearing a face mask. ${ }^{27}$ We classify an individual as engaging in protective behaviour if she/he reports having done both during the recall period. Finally, social-distancing behaviours include activities that reflect individuals' voluntary choices to gather for social reasons: visiting other households and having visitors over to one's own household. Respondents 
are classified as 'social distancing' if they do not report either of these behaviours. If data for a given outcome are missing, for example, because the respondent refused to answer the question, the observation is dropped from the relevant regression.

We acknowledge that the lines between these categories are not always clear; the purpose for each behaviour was not explicitly stated, except for the question about work travel. Therefore, what we classify as market distancing may actually reflect social distancing and vice versa. To address this concern, we show that our main results are robust to recategorising some of the more ambiguous behaviours (attending 10+ person gatherings and having close contact with non-household members) either in the social-distancing or market-distancing indicator (see online supplemental table S2).

Another potential concern is that fulfilling the criteria of social-distancing or protective behaviours may be more likely because they only encompass two behaviours each, while the market-distancing indicator encompasses four. Online supplemental table S3 shows that our main results are robust to using fractional outcomes rather than binary outcomes. In addition, online supplemental tables S4-S6 provide estimates for each individual behaviour within the protective, market-distancing and social-distancing indicators, respectively.

\section{Weight construction}

Weights were constructed in two steps. First, we created base weights to account for the probability of selection of a household, which is determined by the probability of selection of each LASI-DAD participant and the probability of selection of household members, calculated separately for men and women (as one over the number of adult men and women, respectively). Second, we implemented a raking algorithm to obtain post-stratification weights. For this purpose, we used the following raking factors: gender (male/female) ×age (18-39/40-59/60$69 / 70+$ ), gender xeducation (no school/primary or less/ middle/secondary or higher/graduate) and a rural/ urban indicator. Thus, the final weights allow us to match the sample distributions of these variables with their population counterparts while also reflecting differential probabilities of selection of survey participants. Population benchmark distributions were obtained from the 2011 Indian Census for individuals aged 18 and older.

\section{Empirical approach}

To estimate time trends in COVID-19 protective behaviours, we use ordinary least squares regressions of the following form:

$$
y_{i t}=\beta_{0}+\beta_{1} \text { wave }_{t}+\beta_{2} \text { wave }_{t}+\beta_{3} \text { wave }_{t}+\epsilon_{i t},
$$

where $y_{i t}$ is the distancing outcome for individual $i$ measured at time $t$ and wave ${ }_{t}-$ wave ${ }_{t}$ are survey wave dummies, which identify changes in distancing behaviour relative to Wave 1 .
In addition to this basic equation, we also assess whether our estimates are robust to the inclusion of individual fixed effects using the following specification:

$$
y_{i t}=\beta_{0}+\beta_{1} \text { wave }_{t}+\beta_{2} \text { wave }_{t}+\beta_{3} \text { wave }_{t}+\delta_{i}+\epsilon_{i t} .
$$

Finally, we present results that additionally control for COVID-19 caseloads and the Government Response Index:

$$
\begin{aligned}
y_{i t}= & \beta_{0}+\beta_{1}{\text { wave } 2_{t}+\beta_{2} \text { wave }_{t^{+}}} \\
& \beta_{3} \text { wave } 4+\beta_{4} \text { Caseload }_{d t}+\beta_{5} \text { GovtResp }_{t}+\delta_{i}+\epsilon_{i t},
\end{aligned}
$$

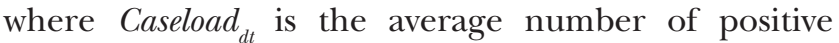
COVID-19 cases reported in the district over the 2 weeks prior to survey date (per 10000 people) and GovtResp is the average value of the Government Response Index in the 2 weeks prior to survey date.

All our equations use sampling weights to ensure our estimates are nationally representative. We cluster SEs at the household level because multiple individuals per household are surveyed in any given wave.

We use the following equation to test for heterogeneity in behaviour outcomes:

$$
y_{i t}=\beta_{0}+\beta_{1} \text { Demo }_{i}+\sum_{k=2}^{4}\left[\beta_{k} \text { Wave }_{k}+\beta_{k+3} \text { Wave }_{k} \times \text { Demo }_{i}\right]+\varepsilon_{i t},
$$

where $y_{i t}$ is one of three behaviour outcomes (marketdistancing, social-distancing or protective behaviours), Wave $_{k}$ is a wave dummy, Demo represents one of four dummy demographic cuts (gender, urbanicity, age older than vs younger than 60 , or highest level of education in the household is primary or less vs middle school or higher). All estimates are weighted, and SEs are clustered at the household level.

\section{RESULTS}

\section{Overall time trends}

Figure 1 shows that initial adherence to protective and social-distancing behaviours was quite high $(89.9 \%$ and $87.7 \%$, respectively), which likely reflects that much of Wave 1 occurred either during or immediately after India's mandatory national lockdown. However, only $37.4 \%$ of individuals reported market distancing during this time, suggesting that most Indians were still engaging in some economic activities during the strictest periods of the lockdown. Figure 1 also highlights declining vigilance over time. Patterns of decline differ in important ways by behaviour type. Protective behaviours, the most stable of the four categories, saw a slight dip in Wave 2 and another in Wave 4 (declining by 3.2 and 4.3 percentage points, respectively). Social distancing, however, has seen significantly larger decreases, with a 30.6 percentage point decline by Wave 4 . Finally, market-based distancing remained essentially steady between Waves 1 and 2, before dropping in Wave 3 . By Wave 4 , only $26.5 \%$ of individuals reported avoiding all the market-based behaviours we measure. 


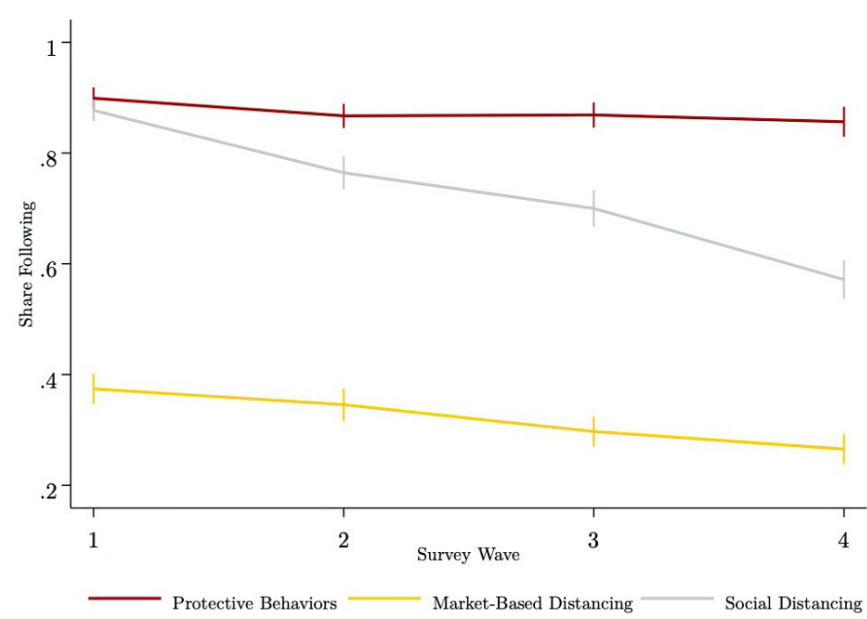

Figure 1 Change in individual behaviour across waves. Notes: Figure depicts regression coefficients of the wave terms from the basic equations as shown in table 1. Data are weighted, and SEs are clustered at the household level. Whiskers denote $95 \% \mathrm{Cls}$. Individuals are considered to be social distancing if they did not report visiting other households or having visitors to their own households. Individuals are considered to be following market-based distancing if they did report any of the following: attended a $10+$ person gathering, had close contact with non-household members, travelled for work or went shopping. Individuals are considered to be engaging in protective behaviours if they report washing their hands and wearing a face mask. 'Don't know' responses and refusals coded to missing.
The first column for each behaviour in table 1 presents results in regression form; weighted differences in behaviour for Waves 2-4 are presented relative to Wave 1. The second column assesses robustness to changes in sample composition by exploiting the panel nature of our data and using within-person variation to identify time trends. If adding fixed effects substantively changes the estimates, this would indicate that individuals who regularly responded to the survey are different from those who sporadically responded, which would raise a concern about sample composition. The final column adds controls for the COVID-19 caseload and the Government Response Index in the previous 2 weeks as a simple way to test whether relaxing behavioural restrictions reflects a shifting disease or policy environment: to the extent that behaviour simply tracks these variables with a lag, controlling for them should attenuate our initial time trend estimates. It is not appropriate to interpret the coefficients on the caseload and policy indicators as causal, however, because the direction of causality is unclear (behaviour could respond to these factors, but both caseloads and policy undoubtedly change in response to behaviour). Moreover, we are not able to control for state and local policy, which may have varied more than the national response during this time. Estimated time trends are generally robust to adding these environmental controls. While time trends in protective behaviour lose statistical significance, these coefficients were small in magnitude initially and do not change

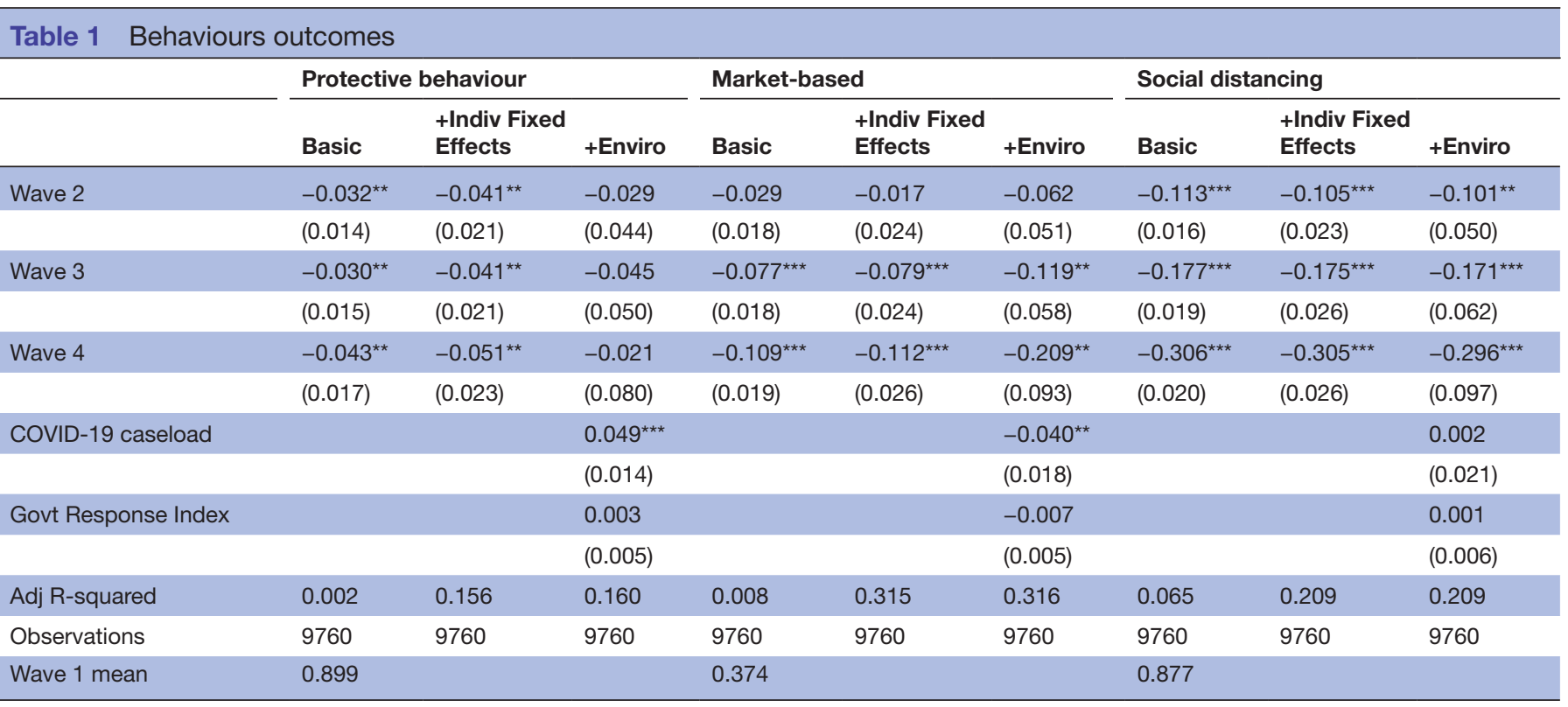

Data are weighted, and SEs are clustered at the household level. COVID-19 caseload is the average number of cases per 10000 in the past 14 days at the district level, except for Assam, Telangana and Delhi, which use state-level caseload due to data constraints. Government Response Index is the 14-day average of the 'overall government response index' from the Oxford Covid-19 Government Response Tracker, with higher values indicating heightened government restrictions. The second and third columns for each outcome include individual fixed effects. Individuals are considered to be social distancing if they did not report visiting other households or having visitors to their own households. Individuals are considered to be following market-based distancing if they did report any of the following: attended a 10+ person gathering, had close contact with non-household members, travelled for work or went shopping. Individuals are considered to be engaging in protective behaviours if they report washing their hands and wearing a face mask. 'Don't know' responses $(n=15)$ and refusals $(n=4)$ coded to missing. Significance is as follows: ${ }^{*} \mathrm{p}=0.1,{ }^{* \star} \mathrm{p}=0.05$ and ${ }^{* \star *} \mathrm{p}=0.01$. 

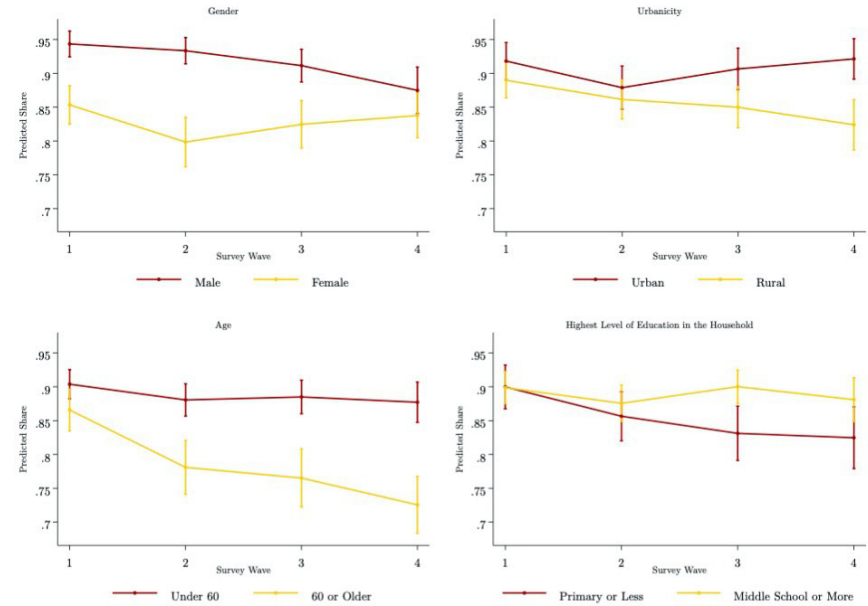

Figure 2 Heterogeneity in protective behaviours across key demographics. Notes: Figures depict the regression coefficients of Wavexdemographic interaction terms. Data are weighted, and SEs are clustered at the household level. Whiskers denote $95 \%$ Cls. Individuals are considered to be engaging in protective behaviours if they report washing their hands and wearing a face mask. 'Don't know' responses and refusals coded to missing.

much. The time trends for social distancing are virtually unchanged, and the decline in market-based distancing becomes even more pronounced. Higher caseloads are associated with more protective behaviour (in line with a behavioural response to underlying disease risk), but less market-based distancing. The latter relationship could reflect increased disease transmission following the reopening of the economy. There is no significant correlation between the Government Response Index and our behavioural measures. We prefer not to overinterpret this result, as this coefficient is identified using within-surveywave variation in the response index-if individuals take time to adjust to shifting government policy, our empirical strategy could understate the import of this variable.

\section{Investigation of disparities}

Vulnerable groups in Indian society are susceptible to disproportionate effects from the pandemic for many reasons: less-educated individuals typically do not hold jobs that can be done remotely, older individuals living with children may not be able to avoid exposure to household visitors and individuals living in densely populated cities may have a more difficult time avoiding contact with others. Behaviour may also vary by gender, given the mobility restrictions and caregiving expectations faced by many Indian women. In this subsection, we quantify how behavioural changes vary based on age, gender, urbanicity and household education.

Figure 2 shows trends in protective behaviour by age (older than vs younger than age 60), urbanicity, gender and highest level of education in the household (primary or less vs middle school or higher). At the beginning of the pandemic (survey Wave 1), we see minimal differences across groups, except that women-who are more likely
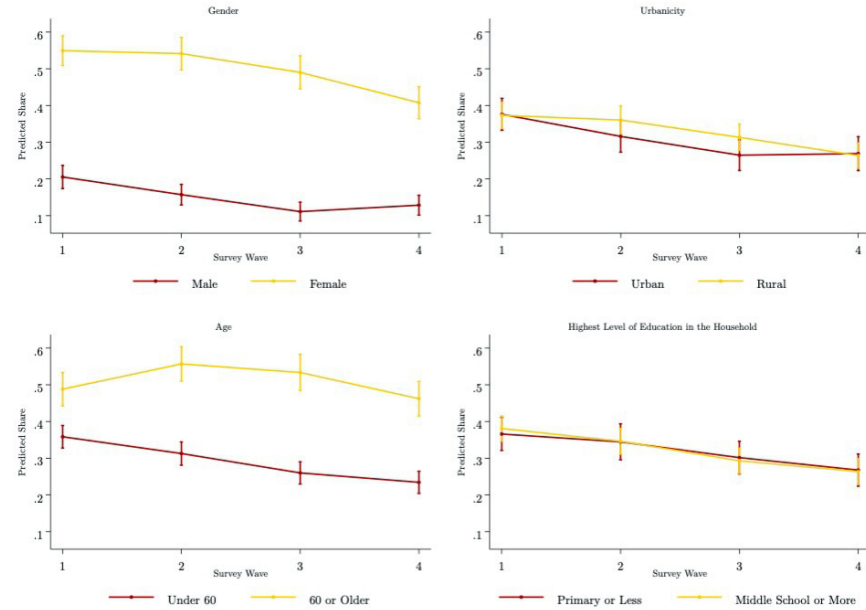

Figure 3 Heterogeneity in market-distancing behaviours across key demographics. Notes: Figures depict the regression coefficients of Wavexdemographic interaction terms. Data are weighted, and SEs are clustered at the household level. Whiskers denote $95 \%$ Cls. Individuals are considered to be market distancing if they did not report any of the following: attended a 10+ person gathering, had close contact with non-household members, travelled for work or went shopping. 'Don't know' responses and refusals coded to missing.

to be homebound due to gender norms-are less likely to report engaging in both protective behaviours. (Consistent with the norms hypothesis, gender differences in handwashing are minimal, while differences in mask wearing are larger and significant.) Adherence among men declines over time, diminishing the gender gap. In contrast, we see a divergence in protective behaviour by age, urbanicity and education. Older individuals $(60+)$ are much more likely to report declining protective behaviour over time, which is worrisome for a cohort that is more vulnerable to severe illness if infected. A decline is also more pronounced among rural dwellers (who have seen persistently lower caseloads) and less educated individuals, signalling higher vulnerability to future waves of infection.

Figure 3 reports trends in market-based distancing by group. During Wave 1, women and older individuals were significantly more likely to report this type of distancing, consistent with their lower levels of economic engagement. In contrast, there is virtually no difference in market-based distancing by urbanicity or education. Gender gaps remain large over time, while age gaps grow in subsequent waves, potentially driven by a return to work among younger cohorts. Finally, figure 4 reports differences in social distancing. We see high levels of social distancing in all groups during Wave 1, which decline significantly over time. Older individuals, women and urban dwellers maintain slightly higher levels of distancing in subsequent survey waves. 

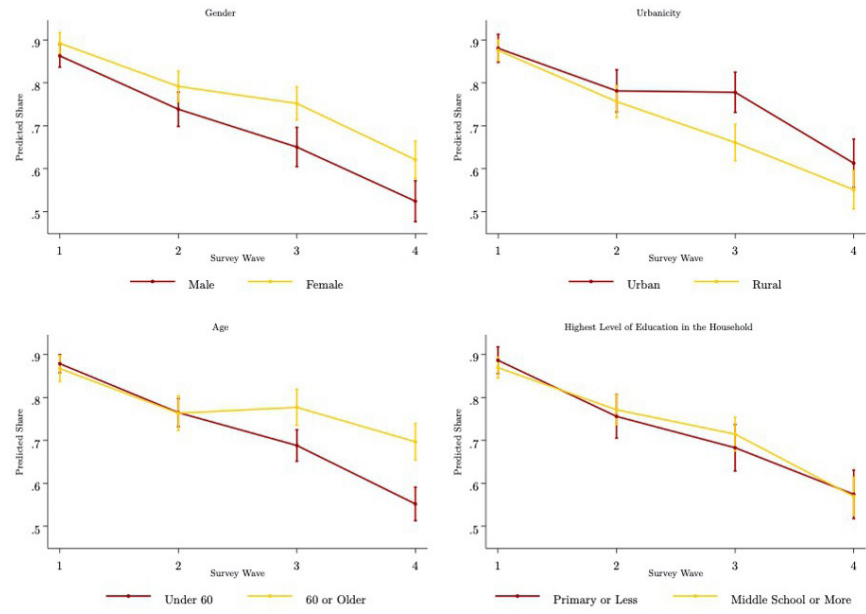

Figure 4 Heterogeneity in social-distancing behaviours across key demographics. Notes: Figures depict the regression coefficients of Wavexdemographic interaction terms. Data are weighted, and SEs are clustered at the household level. Whiskers denote 95\% Cls. Individuals are considered to be social distancing if they did not report visiting other households or having visitors to their own households. 'Don't know' responses and refusals coded to missing.

\section{CONCLUSION}

We find evidence of significant behavioural 'COVID-19 fatigue' in a nationally representative sample of Indian adults. Declines in protective behaviour do not simply reflect an increase in market-based behaviours accompanying India's economic reopening; individuals also increased social contact and (to a lesser extent) reduced mask wearing and handwashing. Our conclusions are unchanged after controlling for local caseload per capita and an index summarising India's nationwide policy response; this suggests that individuals are not just responding to a less risky disease environment or changes in national directives. Rather, restrictive behaviour appears difficult to sustain over time, even conditional on caseloads and policy-although here is it important to keep in mind that caseloads are an imperfect proxy of disease risk, especially in light of concerns about widespread under-reporting, resulting in national statistics that fail to capture true infection and mortality rates. ${ }^{28}$

Another important finding is that declines, especially in social distancing, are found across demographic and socioeconomic groups. Particularly worrying is the significant decline in mask wearing and handwashing among older individuals. While older Indians are less likely to be exposed to others in work or market contexts, their rates of social distancing are like those of the young. Moreover, $69.4 \%$ of the sample live in multigenerational households, where isolating from family members is difficult. Intrahousehold spread is a major contributor to contagion ${ }^{29}$; thus, the steep declines we observe in protective behaviour amount to a 'double risk' for older Indians sharing living quarters and facilities with younger, economically active family members.
One limitation of our research is that it was conducted over the phone. Although mobile phone ownership is high in India, with 93 per cent of households owning a phone according to the nationally representative 20152016 National Family Health Survey, there are significant gaps by gender, wealth and other indicators of socioeconomic status; thus, it is possible that vulnerable households without reliable access to phones may be under-represented in our study. ${ }^{30}{ }^{31}$ Initial evidence also suggests that in India, poorer households have suffered greater economic consequences from the lockdown, ${ }^{32}$ although it is less clear how this would translate into the behaviours measured in our paper; for example, marketdistancing may be less common among phoneless households if they were financially unable to change work behaviour, or market distancing may be more common if this group faced higher rates of job loss. In addition, our binary measures of protective behaviour cannot capture the intensity of adherence (eg, respondents who socially distance half the time would still qualify as social distancers per our definition), which could have significant implications in terms of risk of disease exposure and spread. Finally, while we argue that our observed changes in behaviour are suggestive of growing COVID-19 fatigue, we cannot fully assess the extent to which changes in behaviour reflect personal preferences versus changes in the economic and policy environment as we lack suitable data to completely control for the underlying economic, disease and policy context. For example, reduced marketbased distancing could reflect both the reopening of the economy and businesses, as well as a reduced desire to adhere to protective behaviours.

Generalisability of our findings may be limited due to how varied government responses to the pandemic have been, and particularly how stringent and immediate India's early policy response to COVID-19 was. However, this paper also provides important context in terms of how people were or were not following best practices to reduce disease spread very shortly before March 2021, one of the deadliest outbreaks to-date in India. For example, the decline in protective behaviours we observe could have accelerated disease spread and contributed to the high rates of COVID-19-related morbidity and mortality that started shortly after our last round of data collection. Additional research is needed to rigorously estimate the causal effect of observed behaviour transmission on the trajectory of the pandemic. Additional descriptive research is also essential, as monitoring adherence to distancing guidelines and assessing how public health messaging can be optimised to ensure continued adherence over time will be essential components of India's ongoing battle against COVID-19.

\section{Author affiliations}

${ }^{1}$ Center for Economic and Social Research, University of Southern California, Los Angeles, California, USA

${ }^{2}$ Department of Economics, University of Southern California, Los Angeles, California, USA 
${ }^{3}$ Department of Geriatric Medicine, All India Institute of Medical Sciences, New Delhi, India

${ }^{4}$ Department of Global Health and Population, Harvard TH Chan School of Public Health, Boston, Massachusetts, USA

${ }^{5}$ Department of Hospital Administration, All India Institute of Medical Sciences, New Delhi, India

Acknowledgements We gratefully thank our India-based team of enumerators for their tireless efforts to collect data under challenging conditions; we also owe a debt of gratitude to all survey respondents for graciously taking time to share their experiences during an unprecedented time.

Contributors DB, JL and SS conceptualised, designed and implemented the study and contributed to manuscript preparation. ABD contributed to conceptualisation, design and implementation of the study. NT contributed to data analysis and manuscript preparation. JB and PYK contributed to the study design and managed data collection. SP contributed to project and data management. AA contributed to study design. MA constructed weights for the study and contributed to manuscript preparation. SC and BW contributed to data collection and management. AC, PC and NM contributed to study implementation. JL acts are guarantor.

Funding This research was supported by NIH/NIA U01AG064948 and NIH/NIA 2R01 AG051125.

Competing interests None declared.

Patient consent for publication Not applicable.

Ethics approval This study involves human participants and was approved by USC IRB: UP-20-00277AIIMS, IRB: IEC-300/17.04.2020, RP-29/2020. Participants gave informed consent to participate in the study before taking part.

Provenance and peer review Not commissioned; externally peer reviewed.

Data availability statement Data are available upon reasonable request. Data are available to download via the LASI-DAD website: covid.g2aging.org. Users must sign a data use agreement before being granted access to the data. Note that public release datasets may differ slightly from data used in this paper, which was written with pre-release data. An archived data set which exactly reproduces results in this paper is available upon request.

Supplemental material This content has been supplied by the author(s). It has not been vetted by BMJ Publishing Group Limited (BMJ) and may not have been peer-reviewed. Any opinions or recommendations discussed are solely those of the author(s) and are not endorsed by BMJ. BMJ disclaims all liability and responsibility arising from any reliance placed on the content. Where the content includes any translated material, BMJ does not warrant the accuracy and reliability of the translations (including but not limited to local regulations, clinical guidelines, terminology, drug names and drug dosages), and is not responsible for any error and/or omissions arising from translation and adaptation or otherwise.

Open access This is an open access article distributed in accordance with the Creative Commons Attribution Non Commercial (CC BY-NC 4.0) license, which permits others to distribute, remix, adapt, build upon this work non-commercially, and license their derivative works on different terms, provided the original work is properly cited, appropriate credit is given, any changes made indicated, and the use is non-commercial. See: http://creativecommons.org/licenses/by-nc/4.0/.

\section{ORCID iD}

Simone Schaner http://orcid.org/0000-0001-5722-4265

\section{REFERENCES}

1 Hale T, Angrist N, Goldszmidt R, et al. A global panel database of pandemic policies (Oxford COVID-19 government response Tracker). Nat Hum Behav 2021;5:529-38.

2 Engle S, Stromme J, Zhou A. Staying at home: mobility effects of COVID-19. available at SSRN 3565703, 2020.

3 Nouvellet P, Bhatia S, Cori A, et al. Reduction in mobility and COVID-19 transmission. Nat Commun 2021;12:1-9.

4 Petherick A, Goldszmidt R, Andrade EB, et al. A worldwide assessment of changes in adherence to COVID-19 protective behaviours and hypothesized pandemic fatigue. Nat Hum Behav 2021;5:1145-60.

5 Yilmazkuday H. Stay-at-home works to fight against COVID-19: international evidence from Google mobility data. J Hum Behav Soc Environ 2021;31:210-20.
6 Badr HS, Gardner LM. Limitations of using mobile phone data to model COVID-19 transmission in the USA. Lancet Infect Dis 2021;21:e113.

7 Gatalo O, Tseng K, Hamilton A, et al. Associations between phone mobility data and COVID-19 cases. Lancet Infect Dis 2021;21:e111.

8 Gupta M, Wahl B, Adhikari B, et al. The need for COVID-19 research in low- and middle-income countries. Glob Health Res Policy 2020;5:1-4.

9 Okereke M, Ukor NA, Adebisi YA, et al. Impact of COVID-19 on access to healthcare in low- and middle-income countries: current evidence and future recommendations. Int $J$ Health Plann Manage 2021;36:13-17.

10 Walker PGT, Whittaker C, Watson OJ, et al. The impact of COVID-19 and strategies for mitigation and suppression in low- and middleincome countries. Science 2020;369:413-22.

11 Mullard A. How COVID vaccines are being divvied up around the world. Nature2020;30.

12 Geldsetzer P, Manne-Goehler J, Theilmann M, et al. Diabetes and hypertension in India: a nationally representative study of 1.3 million adults. JAMA Intern Med 2018;178:363-72.

13 Kar SK, Ransing R, Arafat SMY, et al. Second wave of COVID-19 pandemic in India: barriers to effective governmental response. EClinicalMedicine 2021;36:100915.

14 Samarasekera U. India grapples with second wave of COVID-19. Lancet Microbe 2021;2:e238.

15 Kuang J, Ashraf S, Das U, et al. Awareness, risk perception, and stress during the COVID-19 pandemic in communities of Tamil Nadu, India. Int J Environ Res Public Health 2020;17:7177.

16 Yousaf MA, Noreen M, Saleem T, et al. A cross-sectional survey of knowledge, attitude, and practices (KAP) toward pandemic COVID-19 among the general population of Jammu and Kashmir, India. Soc Work Public Health 2020;35:569-78.

17 Pal R, Yadav U, Grover S, et al. Knowledge, attitudes and practices towards COVID-19 among young adults with type 1 diabetes mellitus amid the nationwide lockdown in India: a cross-sectional survey. Diabetes Res Clin Pract 2020;166:108344.

18 Ren Z, Li R, Zhang T, et al. Reduction of human mobility matters during early COVID-19 outbreaks: evidence from India, Japan and China. Int J Environ Res Public Health 2021;18:2826.

19 Saha J, Barman B, Chouhan P. Lockdown for COVID-19 and its impact on community mobility in India: an analysis of the COVID-19 community mobility reports, 2020. Child Youth Serv Rev 2020;116:105160.

20 Saha J, Chouhan P. Lockdown and unlock for the COVID-19 pandemic and associated residential mobility in India. Int $J$ Infect Dis 2021;104:382-9.

21 Thayer WM, Hasan MZ, Sankhla P, et al. An interrupted time series analysis of the lockdown policies in India: a national-level analysis of COVID-19 incidence. Health Policy Plan 2021;36:620-9.

22 Ranjan R, Sharma A, Verma MK. Characterization of the second wave of COVID-19 in India. medRxiv preprint.

23 Lee J, Khobragade PY, Banerjee J. Design and methodology of the longitudinal aging study in India-Diagnostic assessment of dementia (LASI-DAD). J Am Geriatr Soc 2020;68:S5-10.

24 Bhattacharyya R, Bhaduri R, Kundu R. Reconciling epidemiological models with misclassified Case-Counts for SARS-CoV-2 with seroprevalence surveys: a case study in Delhi, India. MedRxiv2020.

25 Malani A, Shah D, Kang G, et al. Seroprevalence of SARS-CoV-2 in slums versus non-slums in Mumbai, India. Lancet Glob Health 2021;9:e110-1.

26 Hale T., N, Angrist EC-B, et al. Variation in government responses to COVID-19. Blavatnik School of Government Working Paper BSGWP-2020/032 Version 7.0, 2020.

27 Honein MA, Christie A, Rose DA, et al. Summary of guidance for public health strategies to address high levels of community transmission of SARS-CoV-2 and related deaths, December 2020. MMWR Morb Mortal Wkly Rep 2020;69:1860-7.

28 Chatterjee P. Is India missing COVID-19 deaths? The Lancet 2020;396:657.

29 Madewell ZJ, Yang Y, Longini IM, et al. Household transmission of SARS-COV-2: a systematic review and meta-analysis. JAMA Netw Open 2020;3:e2031756.

30 International Institute for Population Sciences (IIPS) and ICF. National family health survey (NFHS-4), 2015-2016. India. Mumbai: IIPS, 2017.

31 Barboni G, Field E, Pande R. A tough call: Understanding barriers to and impacts of women's mobile phone adoption in India. Cambridge, MA: Evidence for Policy Design, Harvard Kennedy School, 2018. http://www.tinyurl.com/y49xt26n

32 Gupta A, Zhu H, Doan MK, et al. Economic impacts of the COVID-19 Lockdown in a Remittance-Dependent region. Am J Agric Econ 2021;103:466-85. 